\title{
Avaliação Nutricional em Crianças de uma Creche em Minas Gerais: Relato de Experiência
}

\author{
Fernanda Penido Matozinhos ${ }^{1}$, Manoel Messias Pacheco de Freitas ${ }^{2}$ \\ Glaucia Tatiane Pontes Pascoal ${ }^{3}$, Juliana Oliveira Santos ${ }^{4}$, Lilian Ismenia de Jesus ${ }^{5}$ \\ Suely Medeiros dos Santos ${ }^{6}$, Valeria Aparecida da Silva ${ }^{7}$, Larissa Cristina Silva Bastos ${ }^{8}$
}

\begin{abstract}
RESUMO
Os problemas nutricionais são, indubitavelmente, um dos mais importantes problemas de saúde pública mundialmente; estão diretamente ligados às condições socioeconômicas e culturais menos favorecidas e têm como sua maior vítima a criança. Neste contexto, cabe ressaltar que uma das maneiras de prevenir, diagnosticar e tratar os casos de morbidades infantis se caracteriza por ações que visem à melhora e controle de possíveis distúrbios alimentares. Uma dessas ações refere-se à avaliação nutricional. Assim, o objetivo deste estudo é relatar a experiência de uma avaliação do estado nutricional, por meio de avaliação antropométrica, de crianças de 1 a 5 anos matriculadas em uma creche escolar localizada em um município da região metropolitana de Belo Horizonte, Minas Gerais, além das ações educativas propostas para as crianças e educadores ante ao cenário encontrado. A amostra da pesquisa foi de 62 crianças e as variáveis de estudo foram o sexo, a idade, o peso $(\mathrm{kg})$, a estatura/altura (cm) e o Indice de Massa Corporal (IMC) $\left(\mathrm{Kg} / \mathrm{m}^{2}\right)$. Inicialmente foram realizadas ações de educação em saúde e, posteriormente, realizou-se a aferição de peso e estatura/altura das crianças. Os valores de IMC na criança foram introduzidos no gráfico de IMC infantil. Os dados obtidos foram processados e analisados com auxílio do programa Statistical Software (Stata). Na amostra do estudo observou-se que a tendência de distúrbio nutricional de baixo peso ainda é mais agravante que o excesso de peso. Este estudo demonstrou que alguns avanços são verificados, mas o baixo peso e excesso de peso são problemas relevantes no campo da atenção infantil. A atenção à saúde da criança é uma prioridade dentro dos cuidados à saúde das populações, e a inserção no ambiente infantil nos fez refletir sobre a necessidade de melhor atenção em relação aos futuros adultos do nosso país. Palavras-chave: Avaliação nutricional. Antropometria. Saúde da criança. Enfermagem.
\end{abstract}

\section{NUTRITIONAL ASSESSMENT IN CHILDREN OF A KIDS NURSERY IN MINAS GERAIS: EXPERIENCE REPORT}

ABSTRACT

The nutritional problems are, undoubtedly, one of the most important public health problems worldwide, are directly related to socioeconomic conditions and cultural underprivileged and have as their biggest victim, the child. In this context, it is worth noting that one of the ways to prevent, diagnose and treat cases of child morbidity is characterized by actions that aim to improve and control of possible eating disorders. One of these actions refers to nutritional assessment. Thus, this article describes the experience carried out with children aged 1 to 5 years of age enrolled in a nursery schools located in a municipality in the metropolitan region of Belo Horizonte, Minas Gerais. The goal of the experiment and study was to report the experience of an assessment of the nutritional status, by means of anthropometric values of these children, in addition to educational actions for children and educators outside the scenario encountered.The study was conducted with 62 children and the variables for the same were sex, age, weight $(\mathrm{kg})$, height/height $(\mathrm{cm})$ and body mass index (BMI) $(\mathrm{kg} / \mathrm{m} 2)$. Initially, education activities were carried out in health and then there was the test of weight and height of the children. BMI values in the children were introduced to the children's BMI graphic. The data were processed and analyzed using the Statistical Software program (Stata). This study showed that a trend for underweight disorder is even more aggravating than overweight and also showed that some progress are being made, but the underweight and overweight are major problems in the field of child care. The attention to children's health is a priority within the care for public health care and the inclusion in the child's environment made us reflect on the need for better attention concerning to our country's adults future.

Keywords: Nutrition assessment. Anthropometry. Child health. Nursing.

Recebido em: 4/11/2016

Aceito em: $18 / 2 / 2018$ \footnotetext{
Gerais.nandapenido@hotmail.com

Enfermeiro graduado na Nova Faculdade. enfermagem.novafaculdade@gmail.com

Enfermeira graduada na Nova Faculdade. enfermagem.novafaculdade@gmail.com

Enfermeira graduada na Nova Faculdade. enfermagem.novafaculdade@gmail.com

Enfermeira graduada na Nova Faculdade. enfermagem.novafaculdade@gmail.com

Enfermeira graduada na Nova Faculdade. enfermagem.novafaculdade@gmail.com

Enfermeira graduada na Nova Faculdade. enfermagem.novafaculdade@gmail.com

8 Enfermeira graduada na Escola de Enfermagem da Universidade Federal de Minas Gerais. larissa_bastos94@hotmail.com
}

Enfermeira, doutora em Enfermagem pela Universidade Federal de Minas Gerais e docente da Escola de Enfermagem da Universidade Federal de Minas 


\section{INTRODUÇÃO}

O processo de crescimento e desenvolvimento é dinâmico, contínuo, complexo e segue fases e direções. Além disso, depende da harmonia entre os vários fatores, como os genéticos, nutricionais, hormonais e ambientais (CARDOSO-DEMARTINI et al., 2011).

Por meio da alimentação adequada em quantidade e qualidade, o organismo adquire a energia e os nutrientes necessários para o bom desempenho de suas funções e para a manutenção de um bom estado de saúde. É importante, contudo, conhecer, também, os prejuízos decorrentes do consumo alimentar insuficiente e do consumo alimentar excessivo (ZUCHETTO, 2011).

O tema deste trabalho ganha evidência diante do processo de transição nutricional, enfrentado por inúmeros países da América Latina nos últimos anos, o que implica muitas modificações nos padrões alimentares das populações, como o aumento do consumo de alimentos industrializados. É sabido que tais condições repercutem no desenvolvimento e crescimento infantil (PEÑA; BACALLAO, 2000; POLLA; SCHERER, 2011).

O crescimento no consumo de alimentos com excesso de calorias e baixo teor nutricional pode repercutir, inclusive, no aumento do risco de obesidade na fase adulta e morbimortalidade por Doenças Crônicas Não Transmissíveis (DCNTs) (BRASIL, 2009a; NASCIMENTO; MUNIZ; PINHEIRO, 2010). Autores demonstram que, no Brasil, a evolução do estado nutricional da população é indicativa de importante aumento do excesso de peso, com tendências preocupantes entre as crianças em idade escolar e adolescentes: cerca de $6,9 \%$ e $7,7 \%$ de crianças menores de cinco anos - dos sexos masculino e feminino, respectivamente - estão acometidos por excesso de peso no Brasil. Além disso, a prevalência de excesso de peso em meninos de 5 a 9 anos de idade aumentou de 15,0\%, em 1989, para $34,8 \%$ em 2008-2009. Nas meninas, tendência similar foi observada: $11,9 \%$ para 32,0\% em 2008-2009 (INSTITUTO..., 2010; BRASIL, 2014).

Os problemas nutricionais são, indubitavelmente, dos mais importantes problemas de saúde pública mundialmente, estão diretamente ligados às condições socioeconômicas e culturais menos favorecidas e têm como sua maior vítima a criança (BRASIL, 2009b; DURÁN, 2005; POLLA; SCHERER, 2011; SILVA, 2011).

Neste contexto, cabe ressaltar que uma das maneiras de prevenir, diagnosticar e tratar os casos de morbidades infantis se caracteriza por ações que visem à melhora e controle de possíveis distúrbios alimentares. Uma dessas ações refere-se à avaliação nutricional (SOCIEDADE..., 2009; POLLA; SCHERER, 2011).
Por meio da avaliação nutricional, associada a outras informações - sobre o ambiente em que a criança se insere, por exemplo -, o profissional consegue avaliar a condição geral de saúde de uma criança e investigar se ela está crescendo dentro dos padrões recomendados, possibilitando intervenções efetivas no sentido de evitar, além da morbimortalidade imediata, a persistência da fragilidade do sistema imunológico e a limitação na capacidade de aprendizagem e na socialização (FERREIRA, 2000).

O acompanhamento do crescimento e desenvolvimento é essencial para a avaliação do estado de saúde e nutricional do paciente pediátrico. A antropometria, pela facilidade de execução e baixo custo, tem se revelado o método isolado mais utilizado para o diagnóstico nutricional populacional, principalmente na infância (MIRANDA et al., 2012). A avaliação antropométrica se dá, mais frequentemente, por meio de variáveis, como peso $(\mathrm{kg})$, estatura/altura $(\mathrm{cm})$ e Índice de Massa Corporal (IMC) $\left(\mathrm{Kg} / \mathrm{m}^{2}\right)$.

A assistência à criança deve prever, ainda, a educação em saúde e o preparo dos educadores e da família para o cuidado integral à criança. Assim, avaliar o estado nutricional da população infantil, dentre outros aspectos, traduz-se em apontar caminhos para as transformações necessárias das práticas oferecidas a este grupo.

Diante da relevância e importância do tema e, uma vez que os distúrbios nutricionais afetam gravemente o crescimento, o desenvolvimento e a saúde das crianças, torna-se relevante a busca ativa de crianças em tal condição e posterior encaminhamento para prevenção de possíveis agravos. Assim, o objetivo deste estudo é relatar a experiência de uma avaliação do estado nutricional, por meio de avaliação antropométrica, de crianças de 1 a 5 anos matriculadas em uma creche escolar localizada em um município da região metropolitana de Belo Horizonte, Minas Gerais, além das ações educativas propostas para as crianças e educadores ante o cenário encontrado.

\section{METODOLOGIA}

Trata-se de um estudo descritivo do tipo relato de experiência, desenvolvido em uma creche escolar localizada em um município da região metropolitana de Belo Horizonte, Minas Gerais. Adotou-se uma abordagem quantitativa sustentada na aferição de dados antropométricos, os quais se configuram como fontes para a identificação de distúrbios de crescimento, como desnutrição e excesso de peso. 
Após prévia autorização verbal da direção da Instituição supracitada, uma docente/enfermeira e sete discentes de enfermagem se dirigiram, por três encontros, à creche. Todas as crianças entre um a cinco anos matriculadas no turno vespertino foram convidadas a participar do estudo.

Os dados analisados foram: sexo (variável qualitativa nominal), idade (quantitativa discreta), peso (kg) (quantitativa contínua), estatura/altura $(\mathrm{cm})$ (quantitativa contínua) e IMC $\left(\mathrm{Kg} / \mathrm{m}^{2}\right)$ (qualitativa ordinal). O IMC teve seu uso validado em crianças como bom marcador de adiposidade e sobrepeso, além do fato de que seu valor na infância pode ser preditivo do IMC da vida adulta.

Os acadêmicos de enfermagem foram previamente treinados e realizou-se um estudo prévio sobre alimentação infantil saudável, cálculo e classificação de IMC infantil, escore-z da criança, desnutrição e excesso de peso infantil, embasado em cartilhas do Ministério da Saúde e em artigos pesquisados em bases de dados acessadas eletronicamente: Literatura Latino-Americana em Ciências da Saúde (Lilacs), Scientific Library Online (Scielo).

Inicialmente foram realizadas ações de educação em saúde para as crianças e educadores por meio de palestra. Tais ações enfatizaram a importância da higienização das mãos e como realizá-la, higienização correta dos alimentos e autocuidado, com orientações sobre banho e escovação dentária. Posteriormente explicou-se sobre a importância do consumo nutricionalmente adequado dos alimentos para a manutenção da saúde de acordo com a faixa etária, utilizando-se linguagem clara e objetiva. Ao final da ação promoveu-se uma dinâmica interativa, com o uso de imagens dos alimentos referidos na palestra e cartazes ilustrativos, permitindo, assim, uma melhor compreensão da temática pelas crianças e educadores participantes.

Foram elaboradas planilhas para a inserção das informações antropométricas, visando, deste modo, a assegurar o registro dos dados relevantes à pesquisa. Por fim, realizou-se a aferição de peso e estatura/ altura das crianças, medidas mais sensíveis e específicas para a avaliação do processo de crescimento e desenvolvimento (MIRANDA et al., 2012). A aferição das medidas antropométricas foi realizada em local reservado especialmente para este fim.

$O$ peso foi mensurado com as crianças descalças e com o mínimo de roupas; posicionadas de costas para a balança, com pés juntos e completamente apoiados no centro da plataforma, ombros eretos e olhar na linha do horizonte, de forma que o peso cor- póreo fosse distribuído igualmente em ambos os pés. Após a estabilização da balança faz-se a leitura do peso. Para aferição da estatura/altura, fixou-se uma fita métrica inelástica com divisão de $1 \mathrm{~mm}$ em parede plana, perpendicular ao chão e sem rodapé. Solicitou-se que a criança ficasse em pé, descalça, com os calcanhares, panturrilhas, glúteos e ombros encostados à parede e a cabeça posicionada com o plano de Frankfurt. ${ }^{9}$ Com o auxílio de um esquadro de madeira, deslizou-se sobre a fita métrica até tocar a parte superior da cabeça, registrando a medida da estatura/ altura em centímetros (BRASIL, 2004).

Posteriormente anotou-se os valores obtidos de acordo com o nome, data de nascimento, sexo e idade de cada criança presente na atividade. A identificação das crianças foi realizada com base nas listas de matrículas, obtidas com a diretoria da Instituição. Em seguida, calculou-se o IMC a partir da relação [peso $(\mathrm{kg}) /$ altura $\left.^{2}(\mathrm{~m})\right]$ e os valores de IMC na criança foram introduzidos no gráfico de IMC infantil, que relaciona a idade e o sexo da criança com o valor do IMC. O resultado da relação entre o IMC e a idade indica o escore-z da criança, que varia de acordo com a sua fase de desenvolvimento. Após obter o escore-z da criança, utilizou-se a Classificação do IMC Infantil, para saber se a criança encontrava-se no peso adequado para a sua idade (WORLD..., 2006).

Os dados obtidos foram processados e analisados com o auxílio do Programa Statistical Software (Stata), versão 14.0 (Stata Corp. Texas, USA), e serão apresentados por meio de gráficos.

Todas as educadoras, diretoria e participantes foram informados sobre o objetivo da pesquisa, como a pesquisa seria direcionada e sobre seus direitos como participantes. $O$ consentimento livre e esclarecido se deu por meio do consentimento verbal da diretoria e educadoras, obtido por ocasião dos contatos com os participantes. Ademais, todos os cuidados para tornar os casos não identificáveis foram tomados pelos pesquisadores, evitando a sua identificação e preservando os aspectos éticos do relato de experiência.

\section{RESULTADOS}

O estudo foi realizado com 62 crianças, 30 do sexo masculino e 32 do sexo feminino. Deste total, 2 crianças possuíam 1 ano de idade, 14 apresentavam

\footnotetext{
É um plano estabelecido do ponto mais baixo da margem orbitária ao ponto mais alto da margem do meato acústico externo.
} 
2 anos, 20 delas possuíam 3 anos, 22 tinham 4 anos e 4 delas possuíam 5 anos de idade. Em relação ao gênero, $48,4 \%$ eram do sexo masculino e $51,6 \%$ do sexo feminino. Dentre as faixas etárias avaliadas, a maior prevalência $(67,7 \%)$ foi de crianças com três e quatro anos de idade. As características das crianças estão apresentadas na Figura 1.

Figura 1 - Distribuição da amostra estudada de acordo com a idade e sexo. Região metropolitana de Belo Horizonte, 2014

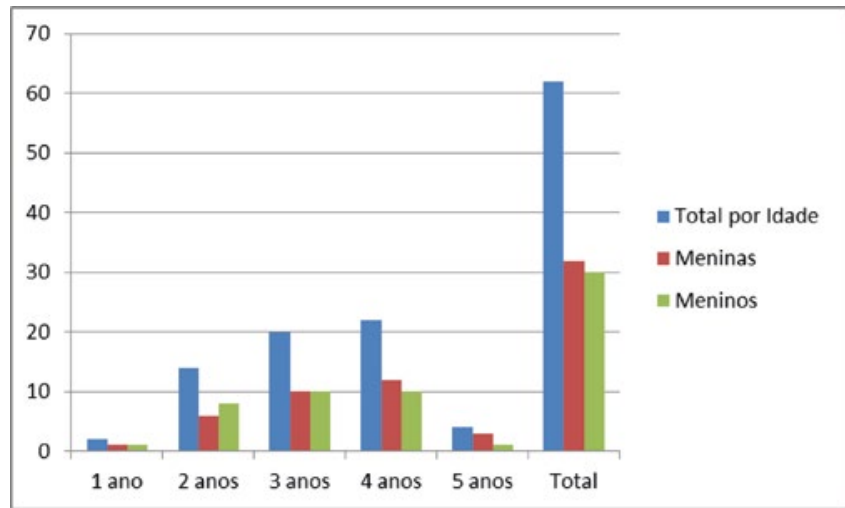

Notas: Eixo $Y=n$; Eixo $X=$ Faixa Etária.

Fonte: Elaborada pelos autores.

Em relação às crianças do sexo feminino, a idade média foi de 42 meses, a idade mínima foi de 22 meses e a máxima de 60 meses. Além disso, o peso médio foi de $17,01 \mathrm{~kg}$ e a estatura/altura média foi de 1,02 m (Figura 2).

Em relação às crianças do sexo masculino, a idade média foi de 40 meses, a idade mínima foi de 22 meses e a máxima de 60 meses. Além disso, o peso médio foi de $16,11 \mathrm{~kg}$ e a estatura/altura média foi de $1,00 \mathrm{~m}$ (Figura 2).

A variável "peso" apresenta maior oscilação quando comparada com a variável "estatura/altura". Observa-se que o peso varia consideravelmente entre as crianças da creche, até mesmo em uma mesma turma, em que as idades são semelhantes.

Figura 2 - Descrição da amostra estudada de acordo com o sexo. Região metropolitana de Belo Horizonte, 2014

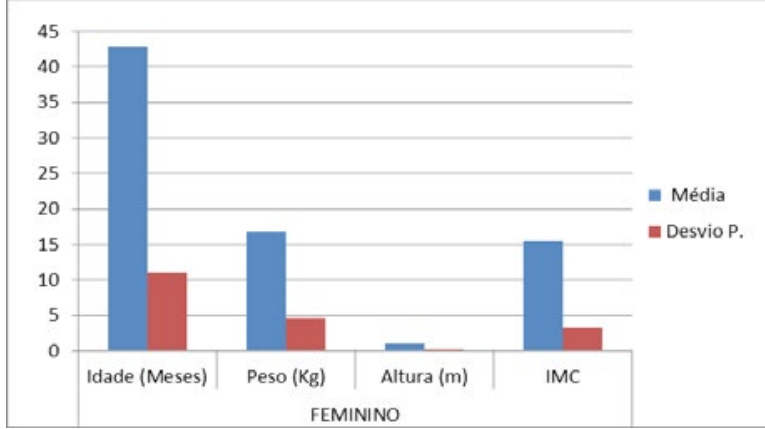

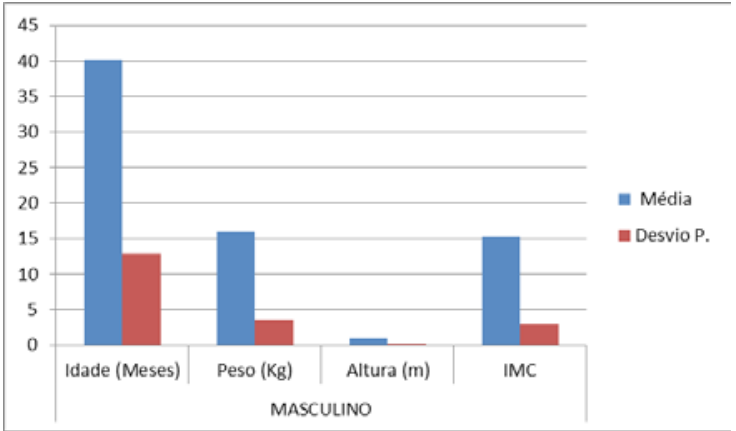

Notas: Desvio P. = Desvio Padrão.

Fonte: Elaborada pelos autores.

Em relação ao estado nutricional das crianças da amostra avaliadas, observou-se que todas as com idade inferior a três anos apresentavam peso adequado para a idade. Nas demais faixas etárias (três, quatro e cinco anos) a maior prevalência foi de estado nutricional adequado para a idade, seguido de baixo peso e peso elevado (excesso de peso) (Figura 3). Neste cenário, observa-se que a tendência de distúrbio nutricional de baixo peso ainda é mais agravante que o excesso de peso.

Figura 3 - Descrição do estado nutricional da amostra estudada de acordo com a idade e sexo. Região metropolitana de Belo Horizonte, 2014

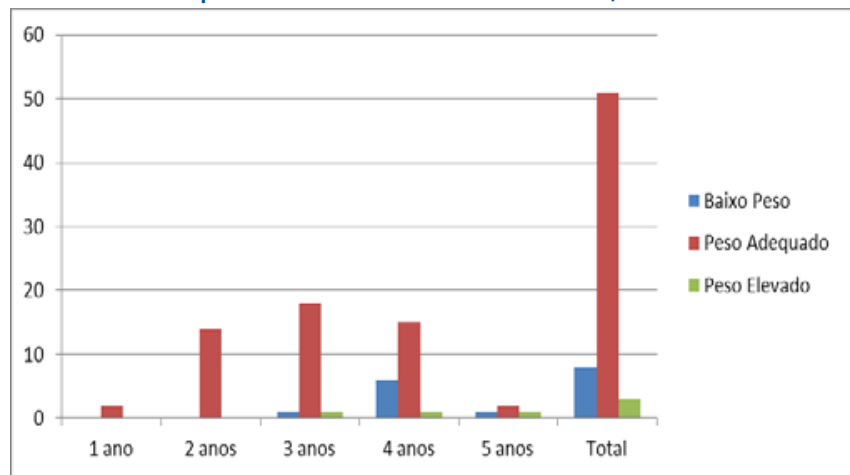

Notas: Eixo $\mathrm{X}=$ Faixa Etária.

Fonte: Elaborada pelos autores.

\section{DISCUSSÃO}

Este relato de experiência demonstrou que o baixo peso e o excesso de peso são problemas relevantes no campo da atenção infantil - resultado também corroborado por pesquisas recentes. Apesar de a alimentação oferecida pela creche ser nutricionalmente equilibrada, nesta amostra o percentual de crianças com baixo peso foi superior ao porcentual de excesso de peso. Dessa forma, é necessária uma maior investigação dos fatores que poderiam levar a tal quadro. 
Hipóteses, como baixa quantidade de alimentos oferecidos às crianças, devem ser consideradas. A educação alimentar de crianças, educadores e familiares é elemento essencial para a construção de hábitos alimentares saudáveis. As oficinas de educação alimentar, adotadas neste estudo, são bons instrumentos de incentivo. A partir dos resultados, é preciso encaminhar essas crianças (tanto as de baixo peso quanto as de excesso de peso) à Unidade Básica de Saúde (UBS) de referência, para acompanhamento tanto pelas Equipes de Saúde da Família como pelos profissionais especializados existentes na UBS.

Ademais, ressalta-se que o governo federal mantém Programas, como o de Saúde na Escola (PSE) e o Nacional de Alimentação Escolar (PNAE), que objetivam contribuir para o crescimento e o desenvolvimento biopsicossocial, além de estimular a formação de hábitos saudáveis e a integração permanente entre educação e saúde, proporcionando melhoria da qualidade de vida da população brasileira.

O PNAE oferece ações de educação alimentar e nutricional aos estudantes (BRASIL, 2009b) e o PSE, por sua vez, contribui para o enfrentamento das vulnerabilidades que comprometem o pleno desenvolvimento de crianças e jovens da rede pública de ensino (BRASIL, 2007). A atenção voltada para as instituições escolares é uma forma de se alcançar melhores resultados para o controle do peso e crescimento, pois é um ambiente ideal para o desenvolvimento de ações coletivas que refletem, inclusive, em ambiente familiar.

Segundo o Ministério da Saúde (2013), o crescimento é um processo dinâmico e contínuo, um dos principais indicadores de saúde na criança. É influenciado por fatores intrínsecos (específicos de cada indivíduo) e fatores extrínsecos (ambientais), entre os quais se destacam a alimentação, a saúde, a higiene, a habitação e os cuidados gerais com a criança. De acordo com Sigulem, Devincenzi e Lessa (2013), a avaliação do crescimento é a medida que melhor define a saúde e o estado nutricional de crianças, pois distúrbios na saúde e nutrição afetam o crescimento infantil. Nos países de baixa e média renda, a maioria dos problemas de saúde e nutrição durante a infância está relacionada com consumo alimentar inadequado e infecções de repetição, posto que essas duas condições estão intimamente relacionadas com o padrão de vida da população, que inclui o acesso à alimentação, moradia e assistência à saúde.
O acompanhamento sistemático do crescimento e do ganho de peso permite a identificação de crianças com maior risco de morbimortalidade, por meio da sinalização precoce da subnutrição e da obesidade (BRASIL, 2014).

O conceito de desenvolvimento, por sua vez, refere-se a uma transformação complexa, contínua, dinâmica e progressiva, que inclui, além do crescimento, maturação, aprendizagem e aspectos psíquicos e sociais. Crescimento e desenvolvimento são aspectos interligados, que influenciam mutuamente a vida do indivíduo.

Neste cenário, destaca-se a avaliação nutricional na infância, que é de extrema importância, pois está diretamente relacionada ao crescimento e desenvolvimento infantil. É, ainda, um indicativo de saúde da população, podendo direcionar o trabalho das equipes de saúde em relação aos problemas influenciados pela alimentação irregular ou inadequada (SIGULEM; DEVINCENZI; LESSA, 2000).

A avaliação nutricional refere-se aos procedimentos desenvolvidos para o diagnóstico individual ou coletivo, visando a identificar o estado nutricional e o potencial de crescimento de pessoas ou grupos de pessoas, bem como monitorar a eficácia das intervenções. Inclui, dentre outros aspectos, a mensuração de medidas antropométricas (ANTONIO et al., 2000).

A vigilância nutricional e o monitoramento do crescimento objetivam promover e proteger a saúde da criança e, portanto, evitar que desvios do crescimento possam comprometer sua saúde atual e sua qualidade de vida futura (BRASIL, 2014).

Um dos melhores métodos de acompanhamento do crescimento infantil é o registro periódico do peso, da estatura e do IMC na Caderneta de Saúde da Criança, que utiliza como parâmetros para avaliação do crescimento de crianças, gráficos, como os de IMC infantil. A inclusão do IMC como parâmetro de avaliação auxilia no exame do estado nutricional de crianças e pode ser preditivo em relação ao IMC na idade adulta (BRASIL, 2014).

Algumas limitações neste trabalho devem ser reconhecidas, dentre elas a avaliação de apenas uma creche. Além disso, a instituição não contava, no momento da coleta dos dados, com informações relevantes, como referentes ao ambiente familiar das crianças. Por fim, é importante ressaltar a dificuldade de comparação dos resultados encontradas neste estudo com os observados por outros autores, em vir- 
tude da diversidade das metodologias aplicadas e de aspectos como o perfil socioeconômico das crianças da amostra.

Apesar dessas potenciais limitações, a experiência vivenciada e os resultados apresentados fornecem evidências de que são imprescindíveis o desenvolvimento de estratégias de intervenção eficazes e a ampliação de programas que contemplem aspectos nutricionais infantis. A avaliação nutricional é fundamental para subsidiar as condutas a serem adotadas para a promoção da saúde e prevenção de agravos, dentre estes as deficiências nutricionais e as DCNTs, como o sobrepeso e o excesso de peso. Em relação às crianças, é imprescindível considerar aspectos, como conceitos adequados de preparo, quantidades ideais das refeições e opções de diversificação alimentar, que contemplem as necessidades nutricionais para cada fase do desenvolvimento (BRASIL, 2009b).

Esses antecedentes e recomendações sugerem a necessidade de mais investigações sobre a atenção à saúde da criança no Brasil, de forma a delinear as prioridades das ações voltadas para tal grupo.

\section{CONSIDERAÇÕES FINAIS}

A atenção à saúde da criança é uma prioridade dentro dos cuidados à saúde das populações. A inserção no ambiente infantil nos fez refletir sobre a necessidade de melhor atenção em relação aos futuros adultos do nosso país. As crianças necessitam de acompanhamento mais rigoroso e atencioso, que envolva pais e educadores, na tentativa de manter o peso adequado e a qualidade de vida para que se tornem adultos saudáveis.

Mesmo após empreendimentos e esforços de órgãos federais, estaduais e municipais, engajados em uma melhoria na qualidade da alimentação escolar, com projetos e programas alimentares, principalmente nas primeiras fases escolares, ainda há deficiência de alguns aspectos, como subsídios, para o sucesso de tais políticas públicas.

Além disso, muitos dos profissionais que trabaIham junto as crianças não demonstram ter qualificação específica necessária para tal. Em algumas vezes, são voluntários sem nenhum vínculo com as áreas de educação e saúde. Dessa forma, destaca-se a importância das ações dos serviços de saúde de forma integrada, com a consequente melhoria na qualidade e na responsabilização da assistência prestada às crianças.

Ficou em nós a conscientização acerca da necessidade de não somente ajudar, mas, sobretudo, enquanto profissionais de enfermagem, nos qualificar e desenvolver práticas e ferramentas para que, junto aos órgãos responsáveis, possamos buscar uma real e significativa melhoria da atual situação das crianças brasileiras.

\section{REFERÊNCIAS}

ANTONIO, Maria A. R. G. M.; MENDES, Roberto T. Estado nutricional e qualidade de vida na criança e no adolescente. Disponível em: <http:www.fef.unicamp.br/fef/sites/uploads/deafa/qvaf/estratégias_cap2.pdf>. Acesso em: 3 mar. 2016.

BRASIL. Ministério da Educação. Decreto no 6.286, de 5 de dezembro de 2007. Institui o Programa Saúde na Escola - PSE. Diário Oficial da União, 6 dez. 2007. Disponível em: <http://portal.mec.gov.br/pnaes/194-secretarias-112877938/secad-educacao-continuada-223369541/14578-programa-saude-nas-escolas>. Acesso em: 1ㅇs. 2017.

. Ministério da Saúde. Pesquisas de orçamentos familiares 2008-2009. Ministério do Planejamento, Orçamento e Gestão. Instituto Brasileiro de Geografia e Estatística IBGE. 2009a.

. Ministério da Saúde. Perspectivas e desafios no cuidado às pessoas com obesidade no SUS: resultados do Laboratório de Inovação no manejo da obesidade nas Redes de Atenção à Saúde. Organização Pan-Americana da Saúde. Brasília: Ministério da Saúde, 2014. 116 p.: il. (Série técnica redes integradas de atenção à saúde, v. 10).

Ministério da Educação. Resolução/FNDE/CD/ $n$. 38 de 16 de julho de 2009. Dispõe sobre o atendimento da alimentação escolar aos alunos da educação básica no Programa Nacional de Alimentação Escolar - PNAE. Brasília, DF: Diário Oficial da União, 2009b. Disponível em: <http:// www.fnde.gov.br/programas/pnae/pnae-sobre-o-programa/pnae-sobre-o-pnae>. Acesso em: 1ㅇs. 2017.

. Ministério da Saúde. Vigilância alimentar e nutricional - Sisvan: orientações básicas para a coleta, processamento, análise de dados e informação em serviços de saúde. Brasília, DF: Ministério da Saúde, 2004.

CARDOSO-DEMARTINI, Adriane de Andre et al. Crescimento de crianças nascidas prematuras. Arq Bras Endocrinol Metab, São Paulo, v. 55, n. 8, p. 534-540, nov. 2011. Disponível em: <http://www.scielo.br/scielo.php?script=sci_arttext\&pid=S0004-27302011000800006\&lng=en\&nrm=iso $>$. Acesso em: 10 jun. 2018. <http://dx.doi.org/10.1590/ S0004-27302011000800006>.

DURÁN, P. Transición epidemiológica nutricional o el efecto mariposa. Arch Argent Pediatr, n. 103, p. 195-197, 2005.

FERREIRA, H. S. Avaliação nutricional de crianças pelo método antropométrico. In: FERREIRA, H. S. Desnutrição - magnitude, significado social e possibilidade de prevenção. Maceió: Edufal, 2000. p. 33-89. V. 2.

INSTITUTO BRASILEIRO DE GEOGRAFIA E ESTATÍSTICA (IBGE). Pesquisa de orçamentos familiares, 2008-2009: antropometria e estado nutricional de crianças, adolescentes e adultos no Brasil. Rio de Janeiro: IBGE, 2010. 
MIRANDA, Marcelle et al. Avaliação antropométrica na infância: uma revisão. Brazilian Journal of Sports Nutrition, v. 1, n. 1, p. 37-45, 2012.

NASCIMENTO, Elisabeth; MUNIZ, Giselia de Santana; PINHEIRO, Isabeli Lins. Evidências da transição nutricional em grupos de escolares entre 7 e 10 anos de idade na cidade de Camaragibe - PE. Revista Brasileira de Ciências da Saúde, v. 14, n. 2, p. 29-36, 2010.

PEÑA, M.; BACALLAO, J. La obesidade en la pobreza: un problema emergente en las Américas. In: ORGANIZACIÓN PANAMERICANA DE LA SALUD (Opas). La obesidad en la pobreza: un nuevo reto para la Salud Publica. Washington, DC: Opas, 2000. p. 3-11.

POLLA, Simone Fátima; SCHERER Fernanda. Perfil alimentar e nutricional de escolares da rede municipal de ensino de um município do interior do Rio Grande do Sul. Caderno de Saúde Coletiva, v. 19, n. 1, p. 111-116, 2011.

SIGULEM, Dirce M.; DEVINCENZI, Macarena U.; LESSA, Angelina $C$. Diagnóstico do estado nutricional da criança e do adolescente. Jornal Pediatria, Rio de Janeiro, v. 76, n. Suppl 3, p. s275-s284, 2000.

SOCIEDADE BRASILEIRA DE PEDIATRIA. Avaliação nutricional da criança e do adolescente - Manual de Orientação/ Sociedade Brasileira de Pediatria. Departamento de Nutrologia. São Paulo: Sociedade Brasileira de Pediatria. Departamento de Nutrologia, 2009. 112 p.

WIDTH, M.; REINHARD, T. Avaliação antropométrica. In: WIDTH, M.; REINHARD, T. MDS: manual de sobrevivência para nutrição clínica. Rio de Janeiro: Guanabara Koogan; 2010. p. 50-52.

WORLD HEALTH ORGANIZATION/WHO. Child Growt Standards. 2006. Disponível em: <http://who.int/childgrowt/ en/>. Acesso em: 3 mar. 2016.

ZUCHETTO, Camila. Estado nutricional, consumo alimentar e atividade física de crianças e adolescentes com Síndrome de Down. 2011. 91 f. Dissertação (Mestrado em Educação física) - Programa de Pós-Graduação em Educação Física, Universidade Federal de Pelotas, Pelotas, RS, 2011. 